\title{
A Common Origin of Neutralino Stars and Supermassive Black Holes
}

\author{
V. I. Dokuchaev, Yu. N. Eroshenko ${ }^{\dagger}$ \\ Institute for Nuclear Research of the Russian Academy of Sciences, Moscow
}

\begin{abstract}
To account for the microlensing events observed in the Galactic halo, Gurevich, Zybin, and Sirota have proposed a model of gravitationally bound, noncompact objects with masses of $\sim(0.01 \div 1) M_{\odot}$. These objects are formed in the expanding Universe from adiabatic density perturbations and consist of weakly interacting particles of dark matter, for example, neutralinos. They assumed the perturbation spectrum on some small scale to have a distinct peak. We show that the existence of this peak would inevitably give rise to a large number of primordial black holes (PBHs) with masses of $\sim 10^{5} M_{\odot}$ at the radiation-dominated evolutionary stage of the Universe. Constraints on the coefficient of nonlinear contraction and on the compactness parameter of noncompact objects were derived from constraints on the PBH number density. We show that noncompact objects can serve as gravitational lenses only at a large $\mathrm{PBH}$ formation threshold, $\delta_{\mathrm{c}}>0.5$, or if noncompact objects are formed from entropic density perturbations.
\end{abstract}

\section{INTRODUCTION}

Dark objects with masses of $\sim(0.01 \div 1) M_{\odot}$ were detected in the Galactic halo when the microlensing of stars from the Large Magellanic Cloud was observed. Brown and white dwarfs and Jupiter-like planets were proposed as possible baryonic candidates. According to the latest data [1], dark objects must account for about $20 \%$ of the hidden mass in the Galactic halo. However, the theory of primordial nucleosynthesis imposes much more stringent constraints on the number of such baryonic objects. Gravitationally bound, noncompact objects, which are also called neutralino stars, were considered among nonbaryonic candidates; these objects can explain some microlensing events with evidence of gravitational lenses being noncompact [2, 3]. The

*dokuchaev@inr.npd.ac.ru

†erosh@ns.ufn.ru 
hypothetical noncompact objects are the lightest objects in the hierarchical large-scale structure of the Universe. In the model of Gurevich, Zybin, and Sirota [3], these are formed immediately after the passage of the Universe to the dust stage.

To reconcile the parameters of noncompact objects with data for the observed microlensing events, Gurevich et al. [3] assumed the cosmological density perturbation spectrum on some small scale to increase sharply with an rms fluctuation of the order of 1 at the peak. For adiabatic perturbations, rms fluctuations of the order of 0.05 correspond to these fluctuations at the radiation-dominated stage on the cosmological horizon scale. As was shown in $[4,5]$, fluctuations with such an rms value give rise to primordial black holes (PBHs) at the radiation-dominated evolutionary stage of the Universe. As we will see below, the mass of the forming PBHs exceeds the mass of noncompact objects by several orders of magnitude. The large difference between the masses of noncompact objects and PBHs stems from the fact that the mass of the radiation contained in a fixed comoving volume at the radiation-dominated stage is much larger than the mass of cold dark matter (CDM) in the same volume at the dust stage.

If the power spectrum of primordial cosmological perturbations is a power law with an index $n>1$, then PBHs are formed in a wide range of masses. If, however, the spectrum has a peak on some scale, then PBHs are formed mostly in a narrow range of masses, near the mass that corresponds to this peak. A sharp peak emerges in the fluctuation spectrum if the inflationary potential $V(\phi)$ has a flat segment $[6,7]$. Indeed, if the derivative at some value of the scalar field is $V^{\prime}=d V(\phi) / d \phi \rightarrow 0$, then a peak emerges in the perturbation spectrum on the corresponding scale, because the mean density perturbation on the horizon scale is $\delta_{\mathrm{H}} \sim M_{\mathrm{Pl}}^{-3} V^{3 / 2} / V^{\prime}$, where $M_{P l}$ is the Planck mass. A similar effect is achieved in inflationary models with several scalar fields $[8$, 9]. In this case, the spectrum outside the peak can have an ordinary shape, for example, it can be a Harrison-Zel'dovich spectrum, and can give rise to galaxies, their clusters and superclusters according to standard scenarios.

Certain evidence for a deviation of the initial perturbation spectrum from a simple power-law shape, more specifically, for a spectral break near large scales, $k \sim(0.06-0.6) h \mathrm{Mpc}^{-1}$, was obtained in the counting of APM galaxies and in the Boomerang and Maxima cosmic microwave background (CMB) anisotropy measurements [10]. Therefore, there is reason to suggest that the spectrum may also exhibit features on small scales.

Here, we show that if the perturbation spectrum has a peak, then there is a clear relationship between the masses of noncompact objects and PBHs; a $\mathrm{PBH}$ mass of the order of $\sim 10^{5} M_{\odot}$ corresponds to a noncompact object mass of the order of $\sim 0.1 M_{\odot}$. For noncompact objects to be able to serve as gravitational lenses, they must originate from sufficiently large dark matter density fluctuations. At the radiation-dominated stage, these fluctuations logarithmically increase with time and become nonlinear even at this stage. To 
study the evolution of the fluctuations at the radiation-dominated stage, we use the nonlinear model proposed by Kolb and Tkachev [11] and specify the initial data for this model according to the linear theory.

Based on standard astrophysical constraints on the $\mathrm{PBH}$ number density in the Universe, we obtained constraints on the rms fluctuations at the peak. In turn, the constraints on the fluctuations give constraints on the radius of a noncompact object. As was shown in [3], a noncompact object can serve as a gravitational lens and can produce observable microlensing events only if its radius exceeds the Einstein radius for this object by no more than a factor of 10. Stringent constraints on the coefficient of nonlinear contraction for noncompact objects follow from this condition.

The inferred relationship between noncompact objects and PBHs holds only for adiabatic cosmological density perturbations. If the density perturbations are entropic, then even if there are large fluctuations in the dust component, the radiation density on the horizon scale is almost uniform and no PBHs are formed.

It should be emphasized that when talking about a common origin of noncompact objects and PBHs, we have in mind not the relationship between individual noncompact objects and $\mathrm{PBHs}$ but the fact that the fluctuations from which the entire collection of noncompact objects and the entire collection of PBHs originate have a common spectrum. If a $\mathrm{PBH}$ emerged at some point in space, then a noncompact object can no longer emerge at this point. Conversely, if there is a noncompact object, then no $\mathrm{PBH}$ was previously (at the radiation-dominated stage) formed at this point.

\section{THE FORMATION OF PBHs}

The PBH formation criterion was analytically derived by Carr [5] and confirmed by numerical calculations $[12,13]$. The region of space with a density $\rho>\rho_{\mathrm{c}}=3 H^{2} / 8 \pi G$ can be roughly considered to be part of the closed Universe. Gravitational collapse of this region and the formation of a $\mathrm{PBH}$ take place if the relative radiation density fluctuation $\delta_{\mathrm{H}}=\left(\rho-\rho_{\mathrm{c}}\right) / \rho_{\mathrm{c}}$ at the time it goes under the horizon satisfies the conditions

$$
\delta_{\mathrm{c}} \leq \delta_{\mathrm{H}} \leq 1
$$

where $\delta_{\mathrm{c}}=1 / 3$. The left-hand inequality implies that the radius of the perturbed region at the time $t$ its expansion stops exceeds the Jeans radius $c t / \sqrt{3}$, while the right-hand inequality corresponds to the formation of a $\mathrm{PBH}$ rather than an isolated universe. The mass of the forming $\mathrm{PBH}$ in this model is

$$
M_{\mathrm{BH}}=\frac{M_{\mathrm{H}}}{3^{3 / 2}}
$$

where $M_{\mathrm{H}}$ is the mass within the horizon. 
In recent years, numerical experiments have revealed the so-called critical gravitational collapse, during which the mass of the forming $\mathrm{PBH}$ is $[14,15]$

$$
M_{\mathrm{BH}}=A M_{\mathrm{H}}\left(\delta_{\mathrm{H}}-\delta_{\mathrm{c}}\right)^{\gamma},
$$

where $A \sim 3, \gamma \simeq 0.36$, and $\delta_{\mathrm{c}} \simeq(0.65 \div 0.7)$. The mass (3) can be much smaller than $M_{\mathrm{H}}$. However, as shown in [16], the PBH mass distribution for critical gravitational collapse is concentrated near $M_{\mathrm{BH}} \sim M_{\mathrm{H}}$, and the contribution of low masses to the cosmological PBH density is modest. Here, we consider various cases where $\delta_{\mathrm{c}}$ lies within the range $1 / 3 \leq \delta_{\mathrm{c}} \leq 0.7$.

Following [6-8], we assume that there is a sharp peak in the fluctuation spectrum on some fixed (in comoving coordinates) scale $\xi=r / a(t)$. Since the $\mathrm{PBH}$ formation threshold is large, $\delta_{\mathrm{H}}>1 / 3$, most $\xi$-scale fluctuations do not collapse into a $\mathrm{PBH}$ but are preserved until the passage to the dust stage if the dark matter particle free streaming length is moderately large [3]. The mass $M_{\mathrm{H}}$ within the horizon as a function of the mass $M_{x}$ of the dust component in fluctuations of the same comoving scale can be calculated by using the standard Friedmann equations. Noncompact objects are formed on time scales $t \leq t_{\text {eq }}$, where $t_{\text {eq }}$ is the time of equality between the matter and radiation densities. At this epoch, a flat cosmological model serves as a good approximation:

$$
\begin{aligned}
& a(\eta)=a_{\mathrm{eq}}\left[2 \frac{\eta}{\eta_{*}}+\left(\frac{\eta}{\eta_{*}}\right)^{2}\right] ; \\
& c t=a_{\mathrm{eq}} \eta_{*}\left[\left(\frac{\eta}{\eta_{*}}\right)^{2}+\frac{1}{3}\left(\frac{\eta}{\eta_{*}}\right)^{3}\right],
\end{aligned}
$$

where $\eta_{*}^{-2}=2 \pi G \rho_{\mathrm{eq}} a_{\mathrm{eq}}^{2} / 3 c^{2}, a_{\mathrm{eq}}$ is the scale factor at time $t_{\mathrm{eq}}$,

$$
\rho_{\mathrm{eq}}=\rho_{0}\left(1+z_{\mathrm{eq}}\right)^{3}=3.2 \cdot 10^{-20}\left(\frac{h}{0.6}\right)^{8}\left(\frac{\Omega_{\mathrm{m}}}{0.3}\right)^{4} \mathrm{~g} \mathrm{sm}^{-3} \text {, }
$$

$1+z_{\text {eq }}=2.32 \cdot 10^{4} \Omega_{\mathrm{m}} h^{2}, \rho_{0}=1.9 \cdot 10^{-29} \Omega_{\mathrm{m}} h^{2} \mathrm{~g} \mathrm{sm}^{-3}$ is the present cosmological matter density, and $h$ is the Hubble constant (in units of $100 \mathrm{~km} \mathrm{~s}^{-1} \mathrm{Mpc}^{-1}$ ). We perform our calculations for two cosmological models: the model with the present matter density parameter $\Omega_{\mathrm{m}}=0.3$ and the cosmological term $\Omega_{\Lambda}=1-\Omega_{\mathrm{m}} \simeq 0.7$ and the model without the $\Lambda$ term and with $\Omega_{\mathrm{m}}=1$. The presence of the $\Lambda$ term reduces only to a change in $\rho_{\text {eq }}$; it does not affect the formation of noncompact objects, because the $\Lambda$ term contributes negligibly to the total cosmological density on time scales $t \leq t_{\mathrm{eq}}$.

For $M_{\mathrm{H}}$ and $M_{x}$, we have the expressions

$$
M_{\mathrm{H}}=\frac{4 \pi}{3} \rho_{\mathrm{H}}\left(a\left(\eta_{\mathrm{H}}\right) \xi\right)^{3}, \quad M_{x}=\frac{4 \pi}{3} \rho_{0}\left(a\left(\eta_{0}\right) \xi\right)^{3} .
$$

On the horizon scale, $a\left(\eta_{\mathrm{H}}\right) \xi=2 c t_{\mathrm{H}}$ with $\eta_{\mathrm{H}} \ll \eta_{*}$ and $\rho_{\mathrm{H}}=3 / 32 \pi G t_{\mathrm{H}}^{2}$. The present density is $\rho_{0}=\rho_{\text {eq }}\left(a_{\text {eq }} / a_{0}\right)^{3}$. 
Using (田), we obtain

$$
\begin{gathered}
M_{\mathrm{H}}=\frac{1}{2^{2 / 3}}\left(\frac{3}{2 \pi}\right)^{1 / 6} \frac{M_{x}^{2 / 3} c}{G^{1 / 2} \rho_{\mathrm{eq}}^{1 / 6}}= \\
=1.96 \cdot 10^{5}\left(\frac{M_{x}}{0.1 M_{\odot}}\right)^{2 / 3}\left(\frac{\Omega_{\mathrm{m}}}{0.3}\right)^{-2 / 3}\left(\frac{h}{0.6}\right)^{-4 / 3} M_{\odot}, \\
t_{\mathrm{H}}=\frac{G M_{\mathrm{H}}}{c^{3}}=0.5\left(\frac{M_{\mathrm{H}}}{10^{5} M_{\odot}}\right) \mathrm{s} .
\end{gathered}
$$

Denote the rms density fluctuation $\delta_{\mathrm{H}}$ by $\Delta_{\mathrm{H}} \equiv\left\langle\delta_{\mathrm{H}}^{2}\right\rangle^{1 / 2}$. The fraction of the radiation mass that transformed into PBHs at time $t_{\mathrm{H}}$ is then $[5,8]$

$$
\begin{aligned}
\beta= & \int_{\delta_{\mathrm{c}}}^{1} \frac{d \delta_{\mathrm{H}}}{\sqrt{2 \pi} \Delta_{\mathrm{H}}} \exp \left(-\frac{\delta_{\mathrm{H}}^{2}}{2 \Delta_{\mathrm{H}}^{2}}\right)= \\
& =\frac{1}{2}\left[\operatorname{erf}\left(\frac{1}{\sqrt{2} \Delta_{\mathrm{H}}}\right)-\operatorname{erf}\left(\frac{\delta_{\mathrm{c}}}{\sqrt{2} \Delta_{\mathrm{H}}}\right)\right] \simeq \frac{\Delta_{\mathrm{H}}}{\delta_{\mathrm{c}} \sqrt{2 \pi}} \exp \left(-\frac{\delta_{\mathrm{c}}^{2}}{2 \Delta_{\mathrm{H}}^{2}}\right),
\end{aligned}
$$

where $\operatorname{erf}(x)$ is the error integral. Since, according to [16], the fraction of the collapsing mass of the Universe for critical gravitational collapse is $0.8 \beta$, Eq. (9) for critical collapse is also a good approximation.

Using (17) and (9), we can calculate the cosmological PBH density parameter $\Omega_{\mathrm{BH}}$ at the present time $t_{0}$ :

$$
\begin{aligned}
& \Omega_{\mathrm{BH}}=\frac{\beta}{2^{2 / 3}}\left(\frac{3}{2 \pi}\right)^{1 / 6} \frac{c}{M_{x}^{1 / 3} G^{1 / 2} \rho_{\mathrm{eq}}^{1 / 6}} \simeq \\
& \simeq 3.45 \cdot 10^{5}\left(\frac{M_{x}}{0.1 M_{\odot}}\right)^{-1 / 3}\left(\frac{\Omega_{\mathrm{m}}}{0.3}\right)^{-2 / 3}\left(\frac{h}{0.6}\right)^{-4 / 3} \frac{\Delta_{\mathrm{H}}}{\delta_{\mathrm{c}}} \exp \left\{-\frac{\delta_{\mathrm{c}}^{2}}{2 \Delta_{\mathrm{H}}^{2}}\right\} .
\end{aligned}
$$

To within a factor of order unity, expression (17) can be derived from a simple estimate $M_{\mathrm{H}} \simeq M_{x} a\left(t_{\mathrm{eq}}\right) / a\left(t_{\mathrm{H}}\right) \simeq M_{x}\left(t_{\mathrm{eq}} / t_{\mathrm{H}}\right)^{1 / 2}$, where $t_{\mathrm{H}}=G M_{\mathrm{H}} / c^{3}$ and $t_{\mathrm{eq}} \sim 6 \cdot 10^{10} \mathrm{~s}$ is the completion time of the radiation-dominated stage. In the same way, we can derive (10) from the estimate $\Omega_{\mathrm{BH}} \simeq \beta a\left(t_{\mathrm{eq}}\right) / a\left(t_{\mathrm{H}}\right)$.

$\mathrm{PBH}$ are formed in the tail of the Gaussian fluctuation distribution, while most noncompact objects are formed from rms fluctuations. Therefore, we repeat that not each fluctuation, by any means, from which a noncompact object formed could collapse into a PBH at time $t_{\mathrm{H}}$. 


\section{THE EVOLUTION OF PERTURBATIONS}

The evolution of a radiation density perturbation at the radiation-dominated stage follows the law [17]

$$
\delta_{\mathrm{r}}=x f(x)+\frac{3 x^{2}}{x^{2}+6} \frac{d}{d x} f(x),
$$

where for the growing mode $f(x)=A_{\text {in }} j_{1}(x / \sqrt{3}), j_{1}$ is the spherical Bessel function, $A_{\text {in }}$ is the normalization constant, $x=k \eta$, and $k$ is the comoving perturbation wave vector. The physical perturbation wavelength satisfies the relations

$$
\lambda_{\mathrm{ph}}(\eta)=a(\eta)\left(\frac{2 \pi}{k}\right) ; \quad M_{x}=\frac{4 \pi}{3} \rho_{0}\left(\frac{\lambda_{\mathrm{ph}}\left(t_{0}\right)}{2}\right)^{3} .
$$

On the horizon scale, $\lambda_{\mathrm{ph}} / 2 \simeq 2 c t, x_{\mathrm{H}} \simeq \pi$, and we obtain from (11) $\delta_{\mathrm{r}}=\delta_{\mathrm{H}}=$ $A_{\text {in }} \phi$, where $\phi \simeq 0.817$.

For adiabatic perturbations, the perturbation in nonrelativistic matter at $x \ll 1$ is $\delta=3 \delta_{\mathrm{r}} / 4$. In [17], an analytic solution was found for $\delta$ in the entire interval from $x \ll 1$ to $x \gg 1$ at the radiation-dominated stage. At $x \gg 1$, this solution is

$$
\delta=\frac{3 A_{\text {in }}}{2}\left[\ln \left(\frac{x}{\sqrt{3}}\right)+\gamma_{\mathrm{E}}-\frac{1}{2}\right],
$$

where $\gamma_{\mathrm{E}}-1 / 2 \simeq 0.077$ and $A_{\text {in }}=\delta_{\mathrm{H}} / \phi$ is the same as in (11).

The applicability of (13) is limited to a linear stage, $\delta \ll 1$. When passing to a nonlinear stage, we will use the spherical model from [11]. In this model, the evolution of adiabatic perturbations is described by the equation

$$
y(y+1) \frac{d^{2} b}{d y^{2}}+\left[1+\frac{3}{2} y\right] \frac{d b}{d y}+\frac{1}{2}\left[\frac{1}{b^{2}}-b\right]=0,
$$

where $y=a(\eta) / a_{\text {eq }}$ and the following parametrization was introduced for the radius of the perturbed region:

$$
r=a(\eta) b(\eta) \xi
$$

Here, $\xi$ is the comoving coordinate and $b(\eta)$ allows for the deceleration of cosmological expansion in the region of enhanced density. The quantity $b$ in (15) can be expressed in terms of $\delta$ as

$$
b=(1+\delta)^{-1 / 3} .
$$

This relation means the passage from the Eulerian description of the perturbation evolution (13) to its Lagrangian description (14).

To solve (14) requires specifying $\delta_{\mathrm{i}}$ at some initial $y_{\mathrm{i}}, b_{\mathrm{i}}=\left(1+\delta_{\mathrm{i}}\right)^{-1 / 3}$ according to (16), and the expansion rate $d b / d y$. In [11], Eq. (14) was solved for entropic perturbations when the initial velocity may be disregarded, $d b / d y \simeq 0$. 
In our case of adiabatic perturbations, the initial velocity is large; we specify it according to the solution (13). At $x \gg 1$ and $y \ll 1$, we have

$$
\begin{aligned}
& x=\frac{\pi}{2^{2 / 3}}\left(\frac{3}{2 \pi}\right)^{1 / 6} \frac{y c}{M_{x}^{1 / 3} G^{1 / 2} \rho_{\mathrm{eq}}^{1 / 6}}= \\
& =2.86 \cdot 10^{6} y\left(\frac{\Omega_{\mathrm{m}}}{0.3}\right)^{-2 / 3}\left(\frac{h}{0.6}\right)^{-4 / 3}\left(\frac{M_{x}}{M_{\odot}}\right)^{-1 / 3} .
\end{aligned}
$$

We obtain from (13), (16), and (17)

$$
\left.\frac{d b}{d y}\right|_{y_{\mathrm{i}}}=-\frac{\delta_{\mathrm{H}} b_{\mathrm{i}}^{4}}{2 y_{\mathrm{i}} \phi} .
$$

We solve Eq. (14) numerically. The time $y_{\mathrm{i}}$ must be chosen in such a way that (13) and (14) are simultaneously valid. An optimum choice is the time when $\delta_{\mathrm{i}}=0.2$. A test shows that the results of our calculations change by no more than $15 \%$ for a different choice of $\delta_{\mathrm{i}}$ in the range $0.1-0.3$. Having specified $\delta_{\mathrm{i}}$, we obtain $x_{\mathrm{i}}$ and $y_{\mathrm{i}}$ from (13) and (17). The evolution of $\delta=b^{-3}-1$ is illustrated in Fig. 1.

The cosmological expansion of noncompact objects stops when $d r / d t=0$. According to [11], this is equivalent to

$$
\frac{d b}{d y}=-\frac{b}{y}
$$

Denote $b$ and $y$ at the time the expansion stops by $b_{\max }$ and $y_{\max }$, respectively. The CDM density in noncompact objects at the same time is

$$
\rho_{\max }=\rho_{\mathrm{eq}} y_{\max }^{-3} b_{\max }^{-3}
$$

and, consequently, the radius of the noncompact object at the stoppage time is

$$
R_{\max }=\left(\frac{3 M_{x}}{4 \pi \rho_{\max }}\right)^{1 / 3} .
$$

The numerically calculated dependence of $R_{\max }$ on $\delta_{\mathrm{H}}$ is shown in Fig. 2.

Thus, we have shown how the radius of a noncompact object at the time its cosmological expansion stops can be determined for the specified perturbation $\delta_{\mathrm{H}}$ on the horizon scale.

\section{THE PARAMETERS OF GRAVITATIONAL LENSES}

After its cosmological expansion stops, a noncompact object begins to contract, with its final radius being

$$
R_{x}=\kappa R_{\max }
$$


where $\kappa$ is the coefficient of nonlinear contraction. It is generally believed [18] that after the cosmological expansion of a noncompact object stops, it is virialized, radially contracting by a factor of 2 , i.e., $\kappa=0.5$. According to the theory of gravitational instability, $\kappa \simeq 0.3$ in a multiflow region [3]. At present, the physical processes that could cause a noncompact object to contract to $\kappa<0.3$ are unknown. Therefore, we take $\kappa \simeq 0.3$ as the lower limit.

For a noncompact object to be able to serve as a gravitational lens producing observable microlensing events in the Galactic halo, its radius should not significantly exceed the Einstein radius

$$
R_{\mathrm{E}}=2 \sqrt{G M_{x} d / c^{2}}
$$

where $d \sim 20 \mathrm{kpc}$ for microlensing in the halo. We define $\varepsilon$ as

$$
\varepsilon \equiv 10 \frac{R_{\mathrm{E}}}{R_{x}}
$$

According to calculations [3], the inequality $\varepsilon \geq 1$ must hold. If $\varepsilon<1$, then the theory comes into conflict with observational data on the light curves [3]. One of the microlensing events with a lens mass $M_{x} \simeq 0.02 M_{\odot}$ was studied in detail in [19]. A comparison of the observed and calculated light curves showed that if a noncompact object has no baryonic core at its center, then it must have the size $R_{x}=1.6 \cdot 10^{13} \mathrm{~cm}$ and a compactness parameter (in our notation) $\varepsilon \simeq 19$. In the presence of a baryonic core with a mass of $0.05 M_{x}$, $R_{x}=5.7 \cdot 10^{13} \mathrm{~cm}$ and $\varepsilon \simeq 4.8$. It was noted in [19] that the model of a pointlike lens for this event is also acceptable because of the large observational errors.

We obtain from (21), (23), and (24)

$$
\kappa \varepsilon=10 \frac{R_{\mathrm{E}}}{R_{\max }}=\frac{1.9 \cdot 10^{15} \mathrm{~cm}}{R_{\max }}\left(\frac{M_{x}}{M_{\odot}}\right)^{1 / 2}\left(\frac{d}{20 \mathrm{kpc}}\right)^{1 / 2}
$$

On the other hand, using our calculations (see the preceding section), we derived a relationship between $R_{\max }$ and $\delta_{\mathrm{H}}$; the quantity $\delta_{\mathrm{H}}$ defines the present cosmological PBH density according to Eq. (10). It should be noted that noncompact objects are formed from rms fluctuations. Therefore, $\delta_{\mathrm{H}}$ from the preceding section should be set equal to the rms fluctuation $\delta_{\mathrm{H}}=\Delta_{\mathrm{H}}$. Using our numerical calculations, we derive the dependence of $\Omega_{\mathrm{BH}}$ on the product $\kappa \varepsilon$ (see Figs. 3 and 4 ).

There are several astrophysical constraints on the mass and number of PBHs. It follows from a constraint on the age of the Universe that $\Omega_{\mathrm{BH}} \leq 1$. If PBHs provide the dominant part of dark matter in the Galaxy, then they must tidally interact with globular clusters by disrupting them. The PBH mass was constrained for this case in [20], $M_{\mathrm{BH}} \leq 10^{4} M_{\odot}$. At $\Omega_{\mathrm{BH}} \sim 1$, PBHs 
are capable of distorting the CMB spectrum if they are formed about $1 \mathrm{~s}$ after the annihilation of $e^{+} e^{-}$- pairs [5]. Mass accretion by black holes at the pregalactic and present epochs contributes to the background radiation in different wavelength ranges. However, calculations strongly depend on the model and yield $\Omega_{\mathrm{BH}} \leq 10^{-3} \div 10^{-1}$ for $M_{\mathrm{BH}} \sim 10^{5} M_{\odot}$. In [22], the constraint $\Omega_{\mathrm{BH}}<0.1$ on intergalactic PBHs was obtained from the condition for the absence of reliable gamma-ray-burst lensing events for $10^{5} M_{\odot}<M_{\mathrm{BH}}<10^{9} M_{\odot}$. A more stringent lensing constraint, $\Omega_{\mathrm{BH}}<0.01$ for the mass range $10^{6} M_{\odot}<M_{\mathrm{BH}}<$ $10^{8} M_{\odot}$, was obtained from VLBI observations of compact radio sources [23].

Let us first consider the microlensing event studied in [19] by assuming that $\varepsilon=0.3$. If the noncompact object has no baryonic core (the vertical line $\kappa \varepsilon \simeq$ 5.7 in Figs. 3 and 4 corresponds to this case), then the constraint $\Omega_{\mathrm{BH}}<0.1$ rules out the interpretation of this event as microlensing by noncompact objects for all $\delta_{\mathrm{c}}=1 / 3 \div 0.7$ in the two cosmological models under consideration. In the presence of a baryonic core (the vertical line $\kappa \varepsilon \simeq 1.44$ ), the case with $\delta_{\mathrm{c}}=0.7$ remains admissible in the cosmological model without the $\Lambda$ term (Fig. 4).

Consider the less stringent condition $\kappa \varepsilon>0.3$, which is satisfied at $\varepsilon>1$ and $\kappa>0.3$. At $\Omega_{\Lambda} \simeq 0.7$ (Fig. 3), the constraint $\Omega_{\mathrm{BH}}<0.1$ rules out all models with $\delta_{\mathrm{c}}<0.5$. At $\Omega_{\mathrm{BH}}>10^{-6}$, the case with $\delta_{\mathrm{c}} \simeq 0.7$ remain possible. If $\Omega_{\Lambda}=0$, then the constraint $\Omega_{\mathrm{BH}}<0.1$ leaves a narrow region (see Fig. 4) with $M_{x}>0.1 M_{\odot}$ and $\delta_{\mathrm{c}}=1 / 3$. The constraint $\Omega_{\mathrm{BH}}<10^{-3}$ completely rules out the models with $\delta_{\mathrm{c}}=1 / 3$, but allows the cases with $\delta_{\mathrm{c}}>1 / 3$.

\section{CONCLUSIONS}

We have shown that the formation of noncompact dark-matter objects (neutralino stars) proposed in $[2,3]$ to account for the observed microlensing events in the Galactic halo must be preceded by the formation of a $\mathrm{PBH}$ with a mass of the order of $\sim 10^{5} M_{\odot}$. These PBHs and neutralino stars are formed from the same peak in the primordial fluctuation spectrum.

Astrophysical constraints on the number of PBHs in the Universe allowed us to constrain the coefficient of nonlinear contraction and compactness parameter for a noncompact object $\kappa \varepsilon$; our constraints are shown in Figs. 3 and 4. The most stringent constraints are obtained in the presence of a cosmological term $\Omega_{\Lambda} \simeq 0.7$. In this case, noncompact objects can serve as gravitational lenses only at a large PBH formation threshold, $\delta_{\mathrm{c}}>0.5$, which was calculated in the model of critical gravitational collapse. At smaller $\delta_{\mathrm{c}}$, the model of noncompact objects as microlensing objects is ruled out. The constraint on $\kappa \varepsilon$ is significantly relaxed if the $\Lambda$ term is small (Fig. 4). However, this possibility is currently considered to be unlikely.

It is important to note that to avoid the situation where $\ln \left(\Omega_{\mathrm{BH}}\right) \ll 0$ requires an accurate adjustment of the parameters for the inflationary model 
that ensures that $\Delta_{\mathrm{H}}$ is in a narrow interval, $\simeq 0.05 \div 0.12$. Therefore, if the observed microlensing events are actually produced by noncompact objects, then these objects most likely originate from entropic density perturbations. In this case, our constraints are removed, as discussed in the Introduction.

The constraints can also be significantly relaxed if for some reason, PBHs are formed in smaller quantities than that given by expression (9). This is possible, for example, in the case of great importance of the nonlinear effects that accompany the generation of metric perturbations at the inflationary stage if these effects suppress the appearance of large fluctuations [24]. Note, however, that the role of nonlinear effects is presently not completely understood, and the results of calculations depend on the specific inflationary model. For example, it was found in [25] that nonlinear effects cause the $\mathrm{PBH}$ formation probability to increase, which is directly opposite to the result from [24].

Conversely, if the noncompact nature of lenses will be proven in the future, then for adiabatic density perturbations, this leads us to conclude that a large number of PBHs with masses of the order of $\sim 10^{5} M_{\odot}$ can be formed at the radiation-dominated sage of the Universe. These $\mathrm{PBH}$ must affect the evolution of galaxies and their nuclei. It may well be that one of such PBHs was found by the Chandra space X-ray observatory in the galaxy M 82 [26]. In a separate paper [27], we develop a model for the formation of galaxies through multiple mergers of protogalaxies with condensation centers in the form of massive PBHs. The mergers of galaxies and the growth of central massive black holes at the galactic nuclei take place simultaneously with the formation of galaxies. The recently found correlations between the masses of central black holes and the bulge velocity dispersion have been shown to naturally arise in this scenario.

Since noncompact objects and PBHs originate from a common perturbation spectrum, we can in principle reconstruct the shape of the perturbation spectrum and determine the PBH mass function from the distribution of noncompact objects in mass and radius using a Press-Schechter-type theory [28]. Unfortunately, only a few objects were detected by microlensing, and such a calculation will become possible only in the future when the statistics will improve.

We may consider a situation that, in a sense, is reverse to the situation described previously. According to the hypothesis [7], the dark halo objects responsible for microlensing are $\mathrm{PBHs}$ with masses of the order of $\sim(0.01 \div$ 1) $M_{\odot}$. If elementary particles with a free streaming length $\ll \xi$ comprise the remaining part of the dark matter, then miniclusters will be formed from these particles at the dust stage. Using formula (7), we can immediately estimate the minicluster mass as $\sim 10^{-11} \div 10^{-8} M_{\odot}$. Such masses are possible if the mass of the dark-matter particles exceeds $1 \mathrm{GeV}[3]$. The formation of PBHs with $\Omega_{\mathrm{BH}} \sim 1$ requires rms fluctuations $\Delta_{\mathrm{H}} \simeq 0.06$. At time $t_{\mathrm{eq}}$, fluctuations in the dust component $\delta \sim 1$, which give rise to miniclusters, correspond to them. 


\section{ACKNOWLEDGMENTS}

We thank K.P. Zybin and V.N. Lukash for helpful discussions. This study was supported by the INTAS (grant no. 99-1065) and the Russian Foundation for Basic Research (project nos. 01-02-17829, 00-15-96697, and 00-15-96632).

\section{References}

[1] C. Alcock, R. A. Allsman, D. R. Alves, T. S. Axelrod, A. C. Becker, D. P. Bennett, K. H. Cook, N. Dalal, A. J. Drake, K. C. Freeman, M. Geha, K. Griest, M. J. Lehner, S. L. Marshall, D. Minniti, C. A. Nelson, B. A. Peterson, P. Popowski, M. R. Pratt, P. J. Quinn, C. W. Stubbs, W. Sutherland, A. B. Tomaney, T. Vandehei, and D. Welch, Astrophys. J. 542, $281(2000)$.

[2] A. V. Gurevich and K. P. Zybin, Phys. Lett. A 208, 276 (1995).

[3] A. V. Gurevich, K. P. Zybin and V. A. Sirota, Physics-Uspekhi 40, 869 (1997), E-print archive astro-ph/9801314.

[4] Ya. B. Zel'dovich and I. D. Novikov, Sov. Astron. 10, 602 (1967).

[5] B. J. Carr, Astrophys. J. 201, 1 (1975).

[6] A. A. Starobinsky, JETP Lett. 55, 489 (1992).

[7] P. Ivanov, P. Naselsky, and I. Novicov, Phys. Rev. D 50, 7173 (1994).

[8] J. Yokoyama, E-print archive astro-ph/9509027.

[9] J. Garcia-Bellido, A. Linde, and D. Wands, Phys. Rev. D 54, 6040 (1996).

[10] J. Barriga, E. Gaztanaga, M. G. Santos, S. Sarkar, E-print archive astroph/0011398.

[11] E. W. Kolb, and I. I. Tkachev, Phys. Rev. D 50, 769 (1994).

[12] D. K. Nadezhin, I. D. Novikov and A. G. Polnarev Sov. Astron. 22, 129 (1978).

[13] I. D. Novikov, A. G. Polnarev, A. A. Starobinsky, and Ya. B. Zeldovich, Astron. Astrophys. 80, 104 (1979).

[14] M. W. Choptuik, Phys. Rev. Lett. 70, 9, (1993).

[15] J. C. Niemeyer, E-print archive astro-ph/9806043.

[16] J. Yokoyama, E-print archive astro-ph/9804041. 
[17] C. Schmid, D. J. Schwarz, and P. Widerin, Phys. Rev. D 59, 043517 (1999).

[18] W. C. Saslaw, Gravitational Physics of Stellar and Galactic Systems (Cambridge Univ. Press, Cambridge, 1985).

[19] V. A. Sirota, JETP, 90, 227 (2000).

[20] B. Moore, Astrophys. J. Lett. 413, 93 (1993).

[21] B. J. Carr, MNRAS 189, 123 (1979).

[22] R. J. Nemiroff, G. F. Marani, J. P. Norris, and J. T. Bonnel, Phys. Rev. Lett 86, 580 (2001).

[23] P. N. Wilkinson, D. R. Henstock, I. W. A. Browne, A. G. Polatidis, P. Augusto, A. C. S. Readhead, T. J. Pearson, W. Xu, G. B. Taylor, and R. C. Vermeulen, Phys. Rev. Lett. 86, 584 (2001).

[24] J. S. Bullock, and J. B. Primack, E-print archive astro-ph/9806301.

[25] P. Ivanov, E-print archive astro-ph/9708224.

[26] P. Kaaret, A. H. Prestwich, A. Zezas, S. S. Murray, D. W. Kim, R. E. Kilgard, E. M. Schlegel, and M. J. Ward, E-print archive astro-ph/0009211.

[27] V. I. Dokuchaev and Yu. N. Eroshenko, Astron. Lett. 27, 759 (2001).

[28] E. V. Bugaev, and K. V. Konishchev, E-print archive astro-ph/0103265. 


\section{FIGURE CAPTIONS}

Fig. 1. The evolution of a CDM density perturbation $\delta$. The plot corresponds to the parameters $\delta_{\mathrm{H}}=0.04, M_{x}=0.1 M_{\odot}$, and $\Omega_{\mathrm{m}}=0.3$. The curve was obtained from formula (13) before the point $\left(y_{\mathrm{i}}=3.96 \cdot 10^{-6}, \delta_{\mathrm{i}}=0.2\right)$, and a numerical solution of Eq. (14) was used at $y>y_{\mathrm{i}}$. The cosmological expansion of a noncompact object stops at the radiation-dominated stage at $y=a / a_{\mathrm{eq}} \simeq 0.49$. The dashed line indicates the evolution of $\delta$ according to the linear theory (13).

Fig. 2. Radius $R_{\max }$ of noncompact objects at the time their expansion stops versus perturbation magnitude $\delta_{\mathrm{H}}$ on the horizon scale. Solid curves 1 , 2 , and 3 correspond to the masses of noncompact objects $M_{x}=1 M_{\odot}, 0.1 M_{\odot}$, and $0.01 M_{\odot}$ in the cosmological model with $\Omega_{\mathrm{m}}=0.3$. Dashed lines 4,5 , and 6 were constructed for $\Omega_{\mathrm{m}}=1$ at the same masses.

Fig. 3. PBH density parameter $\Omega_{\mathrm{BH}}$ versus nonlinear contraction coefficient and compactness parameter for noncompact objects $\kappa \varepsilon$ in the model with $\Omega_{\mathrm{m}}=0.3$. Curves $(1,2,3)$ correspond to the masses of noncompact objects $M_{x}=(0.01,0.1,1) M_{\odot}$ at $\delta_{\mathrm{c}}=1 / 3$. Curves $(4,5,6)$ correspond to $\delta_{\mathrm{c}}=1 / 2$ and curves $(7,8,9)$ correspond to $\delta_{\mathrm{c}}=0.7$ for the same masses. The horizontal and vertical lines correspond to the upper observational limits $\Omega_{\mathrm{BH}}=0.1$, $\Omega_{\mathrm{BH}}=10^{-3}$ and to $\kappa \varepsilon=0.3,1.44$, respectively. The admissible regions lie below the horizontal lines and to the right from the vertical lines.

Fig. 4. Same as Fig. 3 for the cosmological model with $\Omega_{\mathrm{m}}=1$. The vertical lines correspond to $\kappa \varepsilon=0.3,1.44$ and 5.7. 


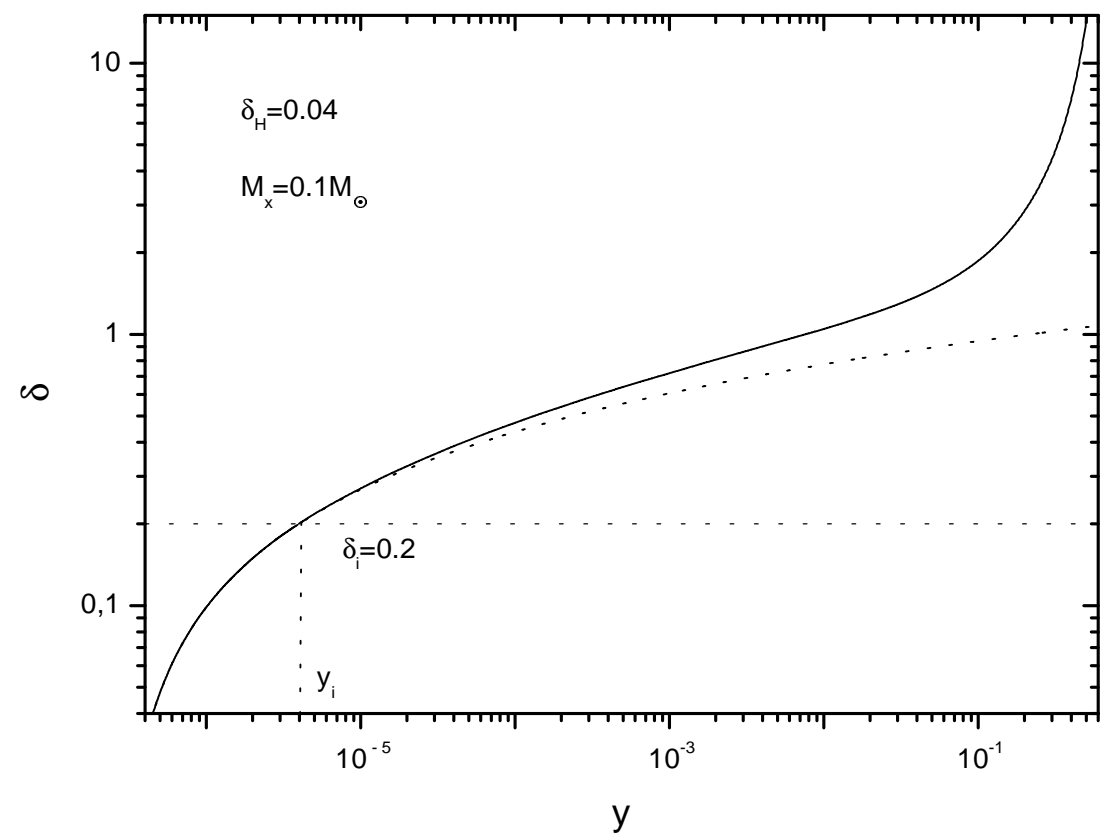




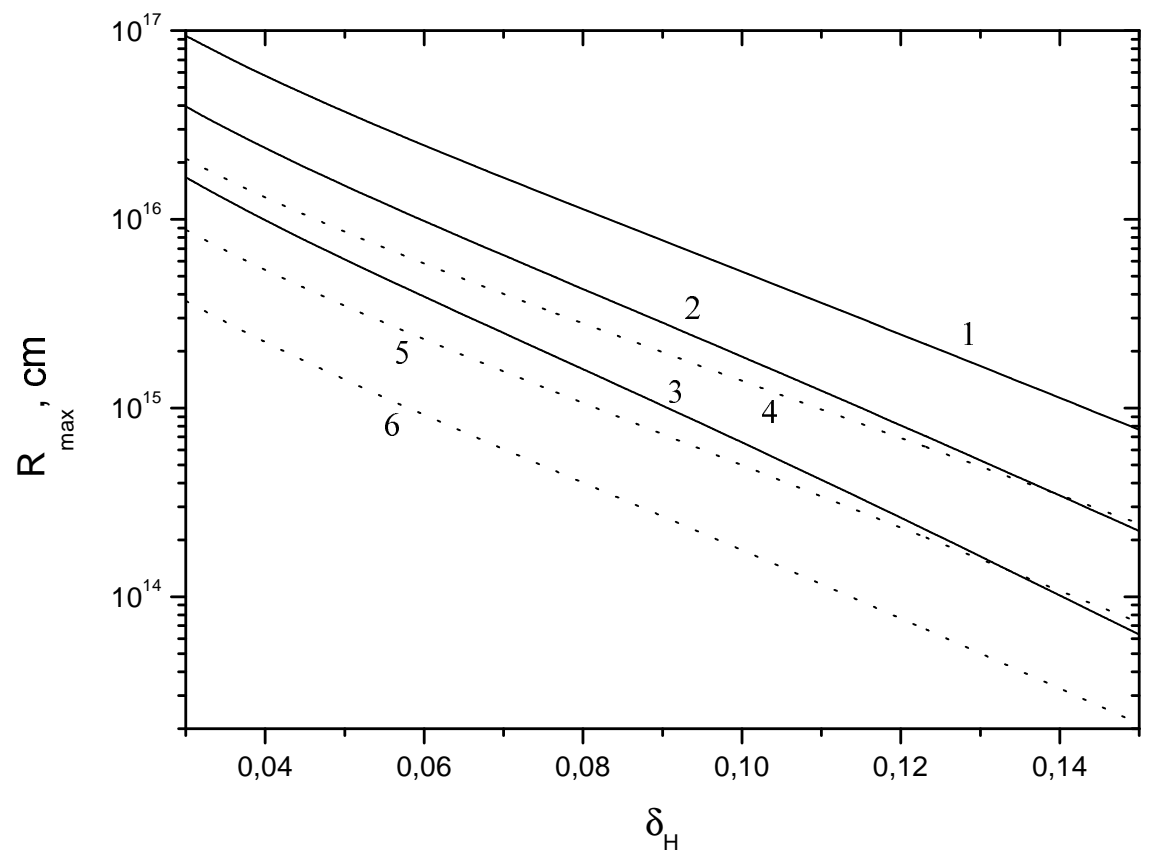




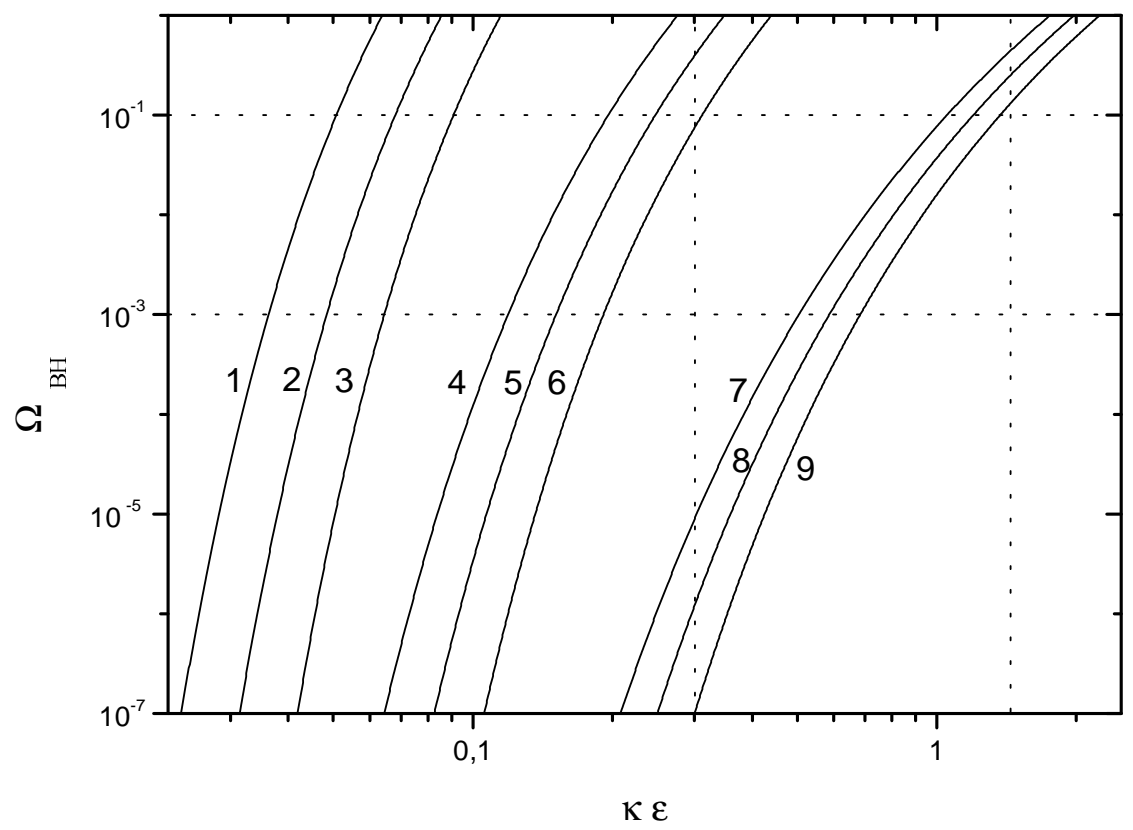




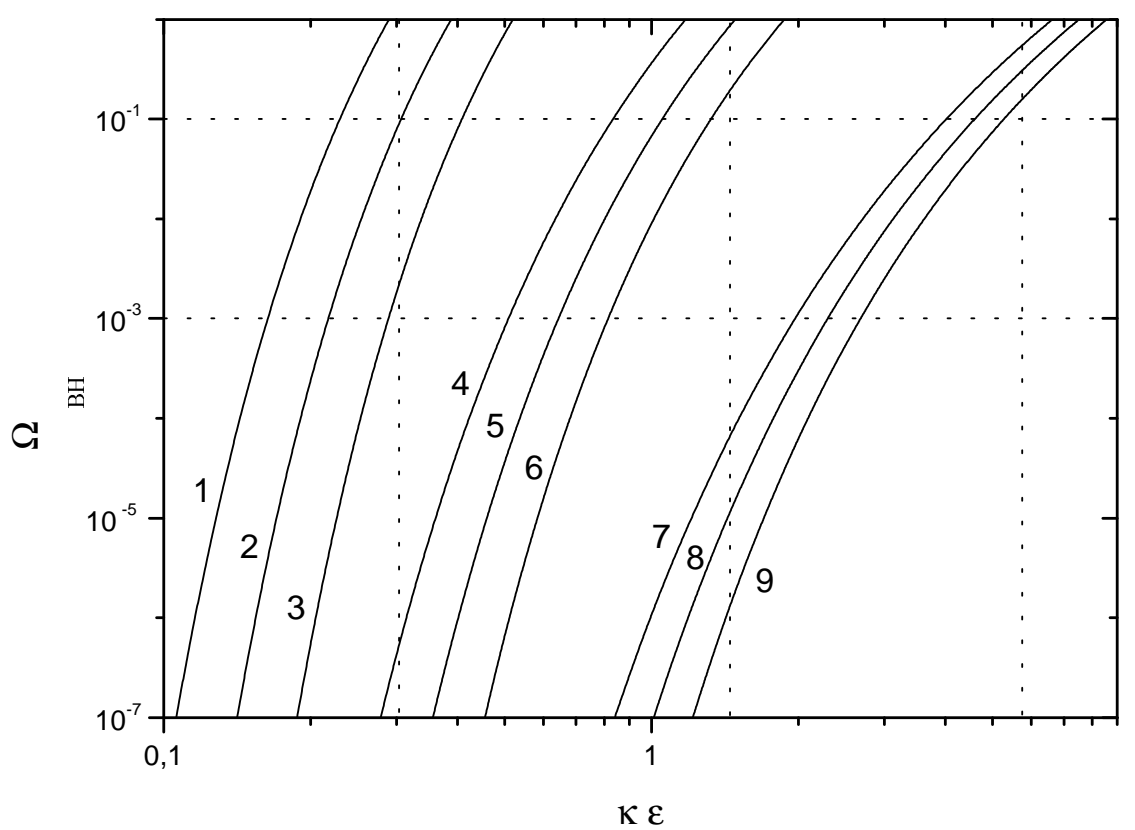

